\title{
Reliability and Validity of a Novel Wearable Device for Measuring Elbow Strength
}

\author{
Marcus Brookshaw ${ }^{1}$, Andrew Sexton ${ }^{2}$ and Chris A. McGibbon ${ }^{2,3, *}$ \\ 1 Department of Mechanical Engineering, University of New Brunswick, Fredericton, NB E3B 5A3, Canada; \\ drakemarcus@gmail.com \\ 2 Institute of Biomedical Engineering, University of New Brunswick, Fredericton, NB E3B 5A3, Canada; \\ asexton@unb.ca \\ 3 Faculty of Kinesiology, University of New Brunswick, Fredericton, NB E3B 5A3, Canada \\ * Correspondence: cmcgibb@unb.ca; Tel.: +1-506-453-4966; Fax: +1-506-453-4827
}

Received: 22 April 2020; Accepted: 9 June 2020; Published: 17 June 2020

check for updates

\begin{abstract}
Muscle strength is an important clinical outcome in rehabilitation and sport medicine, but options are limited to expensive but accurate isokinetic dynamometry (IKD) or inexpensive but less accurate hand-held dynamometers (HHD). A wearable, self-stabilizing, limb strength measurement device (LSMD) was developed to fill the current gap in portable strength measurement devices. The purpose of this study was to evaluate the reliability and validity of the LSMD in healthy adults. Twenty healthy adults were recruited to attend two strength testing sessions where elbow flexor and extensor strength was measured with the LSMD, with HHD and with IKD in random order, by two raters. Outcomes were intra-rater repeatability, inter-rater reproducibility and inter-session reproducibility using intra-class correlation coefficients (ICC). Limits of agreement and weighted least products regression were used to test the validity of the LSMD relative to the criterion standard (IKD), and calibration formulas derived to improve measurement fidelity. ICC values for the LSMD were $>0.90$ for all measures of reliability and for both muscle groups, but over-predicted extensor strength and under-predicted flexor strength. Validity was established by transforming the data with the criterion standard-based calibration. These data indicate that the LSMD is reliable and conditionally valid for quantifying strength of elbow flexors and extensors in a healthy adult population.
\end{abstract}

Keywords: isometric strength; portable strength measurement device; elbow flexors and extensors; repeatability and reproducibility; criterion standard validation

\section{Introduction}

Strength measurements are key outcomes in surgical, clinical and academic research disciplines [1-4]. Valid, reliable strength measurements are essential not only to assessment outcomes [5,6], they often form the basis of clinical decision making in rehabilitation practice [7] and sport medicine [8]. However, options for reliable and accurate objective measurement of muscle strength in the clinic have not changed in three decade and remain limited to hand-held dynamometry (HHD) [9] and isokinetic dynamometry (IKD) [2], both of which have distinct advantages and disadvantages.

HHD is relatively inexpensive, highly portable, and provides an objective, scale measure of isometric strength. Although HHD is reported to be precise enough for clinical assessment $[3,10]$, a detailed review of the literature shows the accuracy of the HHD is limited by the strength [11-14], stability [15,16], and expertise [17,18] of the rater. While IKD may overcome some of these limitations-indeed, it is considered the 'gold standard' for limb strength measurements [2,19-24] and has been shown to have high reliability $[1,2,21,22,25-28]$ — it requires a specialized, stationary 
apparatus that is neither portable nor easily affordable, and requires technical expertise for operation and maintenance.

In this study we propose an alternative approach using a wearable limb strength measurement device (LSMD) [29] for objective strength assessment of elbow flexor and extensor muscle groups [30,31]. The device is described in Figure 1. The LSMD includes an aluminum frame with three members-a long bar with a spring-loaded selection knob at one end (inset Figure 1) connects to a wrist pad with an internal load cell (inset Figure 2) that aligns perpendicular to the wrist. The selection knob has spring-loaded pins that snap into a plate on a third bar with foam pads at either end. Using the knob, the user can select between the extension (Figure 1ii) or flexion (Figure 1iii) configurations for testing limb strength. Alternately, they can lock the device flat for storage/transport (Figure 1i). The portability and ease of use of the LSMD makes it appropriate for a broad spectrum of applications, including: athletics [17,32], physical therapy [33-35], planning and tracking medical interventions [18,36], and academic research [37]. However, the measurement performance of the LSMD has yet to be established.
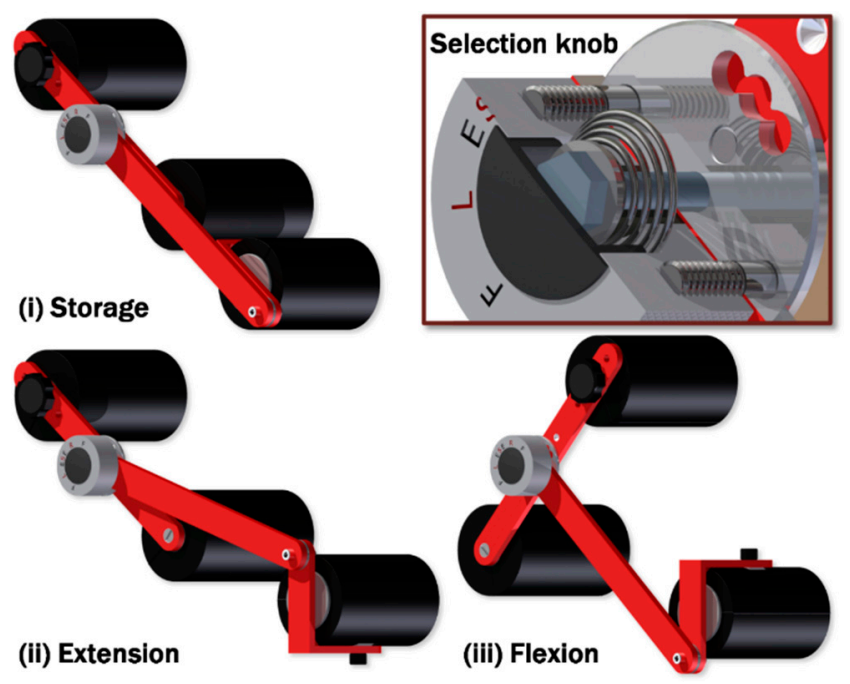

Figure 1. Limb strength measurement device (LSMD) in storage (i), extension (ii), and flexion (iii) configurations. Inset: Spring-loaded selection knob allows user to change between configurations.

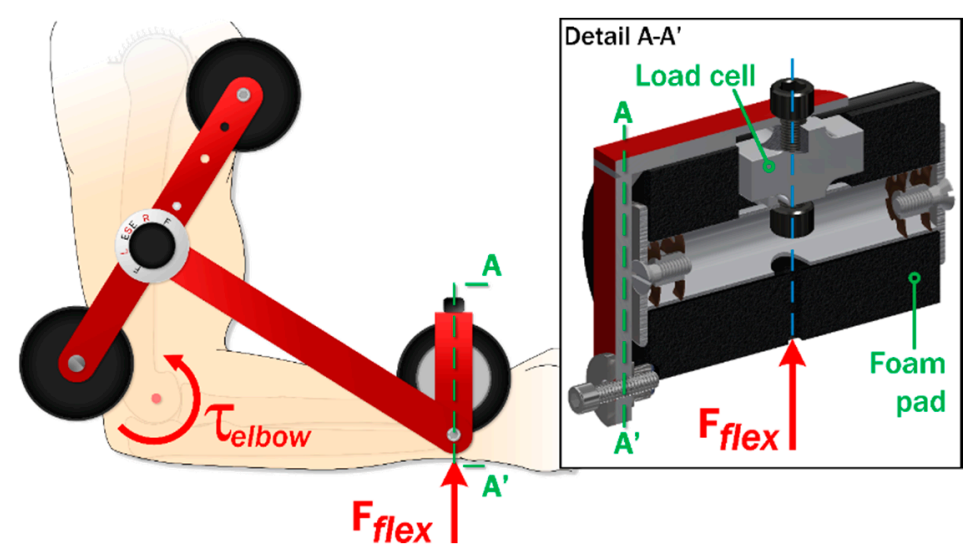

Figure 2. LSMD mode of operation - a wrist pad (Detail A-A') with an integrated load cell is aligned with the wrist of the wearer, allowing the load cell to measure the flexion force $\left(F_{f l e x}\right)$ corresponding to the elbow torque $\left(\tau_{\text {elbow }}\right)$ generated by the wearer. The identical principles apply in extension configuration. 
The purpose of this study was: (1) to evaluate the reliability of the LSMD, in comparison to HHD and IKD performance, and (2) to evaluate the validity the LSMD using IKD as a 'gold standard', and develop correction coefficients for the LSMD to improve performance in both flexor and extensor muscle strength assessment.

\section{Methods}

Evaluation of the LSMD was accomplished using a two-stage experiment comparing isometric strength measurements of the human arm in flexion and extension using the LSMD, the HHD (MicroFET 2 hand-held dynamometer, Hoggan Scientific, LLC, Salt Lake City, UT, USA) and the IKD (Cybex Humac Norm, Computer Sports Medicine, Inc, Stoughton, MA, USA) configured for isometric strength measurement. Specifications for each device are shown below in Table 1.

Table 1. Comparison of specifications for LSMD, Cybex Isokinetic Dynamometer and MicroFET 2 Hand-held Dynamometer.

\begin{tabular}{|c|c|c|c|c|c|}
\hline \multicolumn{2}{|c|}{ Device } & Dimensions (cm) & Mass (kg) & Sensor & Power \\
\hline \multirow{2}{*}{ 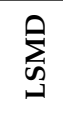 } & Arm & $43 \times 17 \times 10^{+}$ & 1.45 & \multirow{2}{*}{$\begin{array}{l}\text { Linear force: } \\
\text { LC703-300 load cell } \\
\text { (136 kg max capacity) }\end{array}$} & \multirow{2}{*}{$\begin{array}{l}\text { Internally powered: } \\
9 \mathrm{~V} \text { battery }\end{array}$} \\
\hline & Leg & $55 \times 29 \times 10^{+}$ & 2.64 & & \\
\hline \multicolumn{2}{|c|}{ Cybex [38] } & $302 \times 234 \times 152$ & 318 & $\begin{array}{l}\text { Axially aligned torque: } \\
\text { Torque }(678 \mathrm{Nm} \text { max }) \\
\text { and angle }\left(500^{\circ} / \mathrm{s} \text { max }\right)\end{array}$ & $\begin{array}{l}\text { Wall connection: } \\
\text { Isolated, } 20 \text { A } 220 \text { VAC } \\
\text { single-phase line }\end{array}$ \\
\hline \multicolumn{2}{|c|}{ MicroFET 2 [39] } & $10 \times 10 \times 4$ & 0.36 & $\begin{array}{l}\text { Linear force: } \\
\text { Internal load cell (136 } \\
\text { kg max capacity) }\end{array}$ & $\begin{array}{l}\text { Internally powered: } \\
\text { 3.7 V battery } \\
\text { ( } \frac{1}{2} \text { AA cell) }\end{array}$ \\
\hline
\end{tabular}

${ }^{+}$In folded position.

The study was approved by the university research ethics board, and all participants provided informed signed consent prior to enrollment in the study.

\subsection{Participants and Raters}

Twenty healthy adults were recruited from the university student and staff population via poster advertisement and word of mouth. Included were adults between the age of 19 and 60. Exclusion criteria were upper limb fracture or elbow injury in the last 2 years, shoulder or elbow surgery in the last year, or stroke or other neurological disorder affecting the upper limbs.

In addition to the pool of healthy adult participants, a pair of raters was present for all tests, alternating between performing the tests and assisting the other rater, in accordance with the experimental design outlined below. Prior to the first round of testing, both raters received identical training in the use of the HHD, LSMD and IKD from qualified individuals. The two raters were denoted as rater A and rater B. Raters were blind to other raters' assessments.

\subsection{Experimental Design}

A flow diagram of the experimental design is shown in Figure 3. Briefly, the design consisted of two sessions spaced 7-14 days apart. In session I, all 20 participants were assessed with all three devices by rater A. Order of device testing (HHD, LSMD, IKD) was randomized, as was the order of evaluating flexor and extensor strength. Three repetitions were conducted for each device and muscle group. This data was used to evaluate intra-rater reliability. 


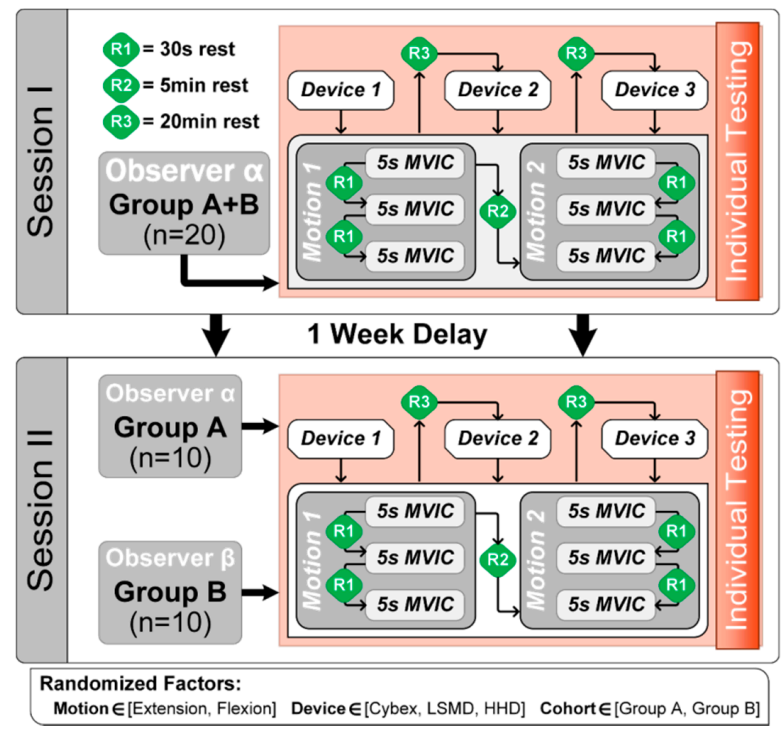

Figure 3. Flow chart of the experimental design.

For session II, participants were randomized into two groups of 10 participants. Group 1 was assessed again by rater A, and group 2 was assessed by rater B. The former data was used to evaluate inter-session reliability, and the latter data used to evaluate inter-rater reliability, for each device and muscle group. Only the dominant arm was tested, determined by asking participants which hand they write with.

\subsection{Protocol}

All tests were conducted at the Andrew and Marjorie McCain Human Performance Lab at the University of New Brunswick. Standard protocols for HHD [40], IKD [41] and LSMD [30,31] were employed for isometric strength testing. Briefly, the elbow was palpated to locate the lateral epicondyle which was designated as the axis of rotation of the elbow joint. The location of the distal forearm load from the testing device was also measured to control for moment arm differences.

Figure 4 shows the positioning of the LSMD on a participant for flexor and extensor strength assessment. The LSMD fixes the joint at approximately 90 degrees flexion. Therefore, the isometric strength tests for HHD and IKD were also conducted at an elbow angle of 90 degrees. This is the typical testing angle for isometric strength measurement of elbow flexors and extensors [40].

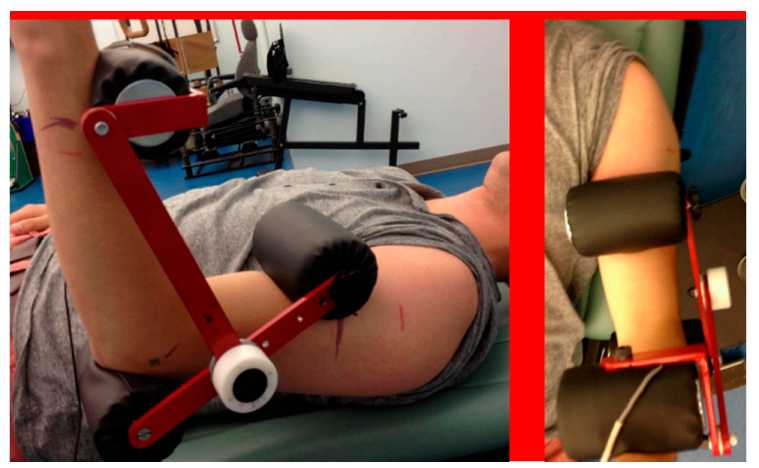

Figure 4. Testing set-up of LSMD in flexion (left) and extension (right).

Maximum voluntary isometric contractions (MVIC) were acquired for each device and muscle group three times each with a $30 \mathrm{~s}$ rest between repetitions. A minimum of $5 \mathrm{~s}$ rest was used between flexor and extensor tests, and a $20 \mathrm{~min}$ break was used between different device tests. The latter was required to ensure muscle recovery from the previous test, and to set-up testing with the other devices. 
To minimize the impact of varied subject motivation and inter-subject variability in related psychological traits, a consistent, positive set of high demand instructions were used to motivate the users throughout their contractions [42].

\subsection{Data Analysis}

HHD and LSMD measure force, whereas the IKD measures torque. To facilitate comparison with the IKD, each MVIC recorded with the HHD and LSMD was converted to an equivalent torque at the elbow using the force measurements and the measured distances between the elbow joint center and line of action of the HHD and LSMD for each participant. As such, muscle strength was measured as a torque expressed in units of $\mathrm{N}^{*} \mathrm{~m}$.

\subsubsection{Objective 1: Reliability of LSMD for Strength Assessment}

The torque data were used to determine two measures of reproducibility (inter-session and inter-rater reliability) and one measure of repeatability (intra-rater reliability), using the intraclass correlation coefficient ICC as described by Shrout and Fleiss [43].

For intra-rater reliability the three repeated measurements were treated as fixed effects in a two-way mixed effects ANOVA model (ICC model 3 for consistency, or ICC component of variance attributable to the targets (the cohort of 20 participants) and those associated with random error. The $\operatorname{ICC}_{\text {con }}(3,1)$ was taken to be a measure of how well the same rater in identical conditions was able to consistently measure the strength of a participant.

For the two measures of reproducibility, each rater was assigned a different randomly selected subset of 10 participants from the full cohort-Group 1 was used to assess inter-session reliability and Group 2 was used to assess inter-rater reliability. Maximum strength from the three repetitions of each session was used in this analysis. ICC model 2 for absolute agreement, or ICC $_{a a}(2,1)$ using a two-way random effects ANOVA model was used to obtain the components of variance attributable to the targets (the cohort of 10 participants), judges ( 2 raters or 2 sessions) and those associated with random error. The $\operatorname{ICC}_{\mathrm{aa}}(2,1)$ was taken to be a measure of the agreement between two different raters and between two different sessions with the same rater.

In addition, a post-hoc analysis using simple main effects of the ANOVA model was used to follow-up on the significance of differences between raters and between sessions.

\subsubsection{Objective 2: Validity of the LSMD for Assessment}

Using the torque data from Session I, the validity of the LSMD was evaluated by directly comparing it to the corresponding IKD data across all 20 participants. This comparison treated IKD as the 'gold standard' for isometric strength measurement, with the relative performance of the LSMD determined both qualitatively using limits of agreement (LoA) [44], and quantitatively, using weighted least products (WLP) regression [45]. The WLP regression was required rather than an ordinary least squares analysis due to the expected, and observed, heteroscedasticity of strength measures [46].

Although the LSMD was built with stiff steel members, the joint assembly and the foam contact rollers allow for small deflections of the device, especially with stronger users, potentially over- or under-estimating the elbow torque being applied. To correct for these effects, the WLP regression analysis between LSMD and the IKD from the above analysis was used to form the coefficients (slope $b$, and intercept $a$ ) of a linear regression equation

$$
\tau_{L S M D}=a+b * \tau_{I K D}
$$

where $\tau_{L S M D}$ and $\tau_{I K D}$ are the measured torques from LSMD and IKD session I data, respectively. The coefficients can then be used to correct the measured LSMD data by calibrating the LSMD against the criterion standard IKD.

$$
\tau_{L S M D}^{C a l}=\frac{1}{b}\left(\tau_{L S M D}-a\right)
$$

where $\tau_{L S M D}^{C a l}$ is the calibrated LSMD data. 
Finally, to test the validity of the calibration, the LSMD data from Session II data were corrected with the derived equation and compared to Session II IKD data using WLP regression as described above.

All statistical analyses were conducted with SPSS (Statistical Package for the Social Sciences, v23, IBM-SPSS, Chicago, IL, USA).

\section{Results}

Twenty healthy adults (eight female) were recruited into the study. There were no drop-outs. Participant demographics and handedness, summarized by group and for the full cohort, are shown in Table 2. Mean peak elbow torques in extension and flexion for all three devices are summarized in Table 3 for each group and for the full cohort.

Table 2. Participant demographics.

\begin{tabular}{cccc}
\hline & Group 1 & Group 2 & Full Cohort \\
\hline Sex n Female/Male & $3 \mathrm{~F} / 7 \mathrm{M}$ & $5 \mathrm{~F} / 5 \mathrm{M}$ & $8 \mathrm{~F} / 12 \mathrm{M}$ \\
Hand dominance n Right/Left & $8 \mathrm{R} / 2 \mathrm{~L}$ & $8 \mathrm{R} / 2 \mathrm{~L}$ & $16 \mathrm{R} / 4 \mathrm{~L}$ \\
Age mean years (s; min-max) & $24(3 ; 21-32)$ & $28(10 ; 21-53)$ & $26(8 ; 21-53)$ \\
Height mean cm (s; min-max) & $171(11 ; 152-188)$ & $172(11 ; 154-192)$ & $172(11 ; 152-192)$ \\
Mass mean kg (s; min-max) & $73(18 ; 48-101)$ & $71(16 ; 55-108)$ & $72(17 ; 48-108)$ \\
BMI mean BMI (s; min-max) & $25(4 ; 19-33)$ & $24(4 ; 19-33)$ & $24(4 ; 19-33)$ \\
\hline \multicolumn{2}{c}{$s$ = Standard deviation; BMI = Body mass index; R = Right; $\mathrm{L}$ = Left; F = Female; M = Male. }
\end{tabular}

Table 3. Peak torque characteristics of each participant group and full cohort.

\begin{tabular}{|c|c|c|c|c|}
\hline & & \multicolumn{3}{|c|}{ Peak Extension $\tau$ mean $(s ; \max -\min )$} \\
\hline & & Group A & Group B & Full Cohort \\
\hline \multirow{2}{*}{ 酎 } & $S I$ & $47.1(20.3 ; 20.6-78.7)$ & $31.2(7.9 ; 19.5-41.8)$ & $39.2(17.0 ; 19.5-78.7)$ \\
\hline & $S I I$ & $50.0(25.5 ; 20.6-94.9)$ & $40.0(10.7 ; 25.0-56.0)$ & $45.0(19.7 ; 20.6-94.9)$ \\
\hline \multirow{2}{*}{$\sum_{\text {H }}^{\theta}$} & SI & $59.0(34.9 ; 19.7-120.2)$ & $41.6(17.7 ; 21.5-77.2)$ & $50.3(28.8 ; 19.7-120.2)$ \\
\hline & S II & $62.8(39.0 ; 19.2-143.9)$ & $43.4(14.5 ; 23.7-65.1)$ & $53.1(30.3 ; 19.2-143.9)$ \\
\hline \multirow{4}{*}{$\underline{\theta}$} & SI & $46.1(22.6 ; 16.5-76.6)$ & $31.8(10.3 ; 18.3-54.5)$ & $39.0(18.6 ; 16.5-76.6)$ \\
\hline & $S I I$ & $47.3(26.1 ; 19.5-93.0)$ & $38.0(10.4 ; 21.5-51.3)$ & $42.6(19.9 ; 19.5-93.0)$ \\
\hline & & \multicolumn{3}{|c|}{ Peak Flexion $\tau$ mean $(s ; \max -\min )$} \\
\hline & & Group A & Group B & Full Cohort \\
\hline \multirow{2}{*}{ 酎 } & $S I$ & $44.4(18.8 ; 16.6-75.5)$ & $40.6(10.5 ; 26.8-59.8)$ & $42.5(14.9 ; 16.6-75.5)$ \\
\hline & S II & $52.6(23.1 ; 21.0-85.2)$ & $38.8(8.7 ; 27.2-54.3)$ & $45.7(18.4 ; 21.0-85.2)$ \\
\hline \multirow{2}{*}{$\sum_{\text {H }}^{\theta}$} & SI & $45.0(19.4 ; 20.8-68.8)$ & $35.7(10.3 ; 19.8-51.3)$ & $40.3(15.9 ; 19.8-68.8)$ \\
\hline & $S I I$ & $49.1(21.1 ; 23.0-77.2)$ & $36.6(10.2 ; 21.7-52.9)$ & $42.9(17.4 ; 21.7-77.2)$ \\
\hline \multirow{2}{*}{ 晃 } & SI & $50.9(23.2 ; 23.4-91.6)$ & $40.3(11.3 ; 24.0-60.4)$ & $45.6(18.6 ; 23.4-91.6)$ \\
\hline & $S I I$ & $55.3(23.3 ; 26.7-87.2)$ & $44.5(13.3 ; 28.1-72.6)$ & $49.9(19.2 ; 26.7-87.2)$ \\
\hline
\end{tabular}

$\mathrm{HHD}=$ = Hand-held dynamometry; LSMD = Limb strength measurement device; IKD = fixed-isokinetic (isometric) dynamometry; $s$ = Standard deviation; $\tau=$ Torque in $\mathrm{N}^{*} \mathrm{~m} ; \mathrm{S} \mathrm{I}=$ Session one testing; $\mathrm{S} \mathrm{II}=$ Session two testing.

\subsection{Reliability Analysis}

\subsubsection{Intra-Rater Repeatability}

This analysis used repeated measurement data ( 3 trials) from session I measured by rater A on the full cohort $(n=20)$. Reliability results using the $\operatorname{ICC}_{\mathrm{con}}(3,1)$ model are shown in Table 4 . All three devices were found to be repeatable with all ICC values exceeding 0.96 except for flexion strength with IKD which was a little lower at 0.915 but still considered in the excellent range. 
Table 4. Reliability results for intra-rater repeatability, inter-rater reproducibility and inter-session reproducibility.

\begin{tabular}{|c|c|c|c|c|c|c|}
\hline & \multicolumn{2}{|c|}{ Extension } & \multicolumn{4}{|c|}{ Flexion } \\
\hline & \multicolumn{3}{|c|}{$\mathrm{CI}_{95}$} & \multicolumn{3}{|c|}{$\mathrm{CI}_{95}$} \\
\hline & ICC & [LB, UB] & $d_{\text {Cohen }}$ & ICC & [LB, UB] & $d_{\text {Cohen }}$ \\
\hline \multicolumn{7}{|c|}{ Intra-rater Repeatability $^{\dagger}$} \\
\hline HHD & 0.975 & {$[0.948,0.989]$} & 15.0 & 0.969 & {$[0.935,0.986]$} & 12.9 \\
\hline LSMD & 0.996 & {$[0.991,0.998]$} & 34.6 & 0.965 & {$[0.924,0.985]$} & 12.5 \\
\hline IKD & 0.975 & {$[0.947,0.990]$} & 15.0 & 0.915 & {$[0.829,0.964]$} & 7.9 \\
\hline \multicolumn{7}{|c|}{ Inter-rater Reproducibility $\ddagger$} \\
\hline HHD & 0.575 & $\begin{array}{c}-0.107, \\
0.891]\end{array}$ & 2.7 & 0.875 & {$[0.597,0.967]$} & 5.1 \\
\hline LSMD & 0.897 & {$[0.653,0.973]$} & 6.8 & 0.856 & {$[0.526,0.962]$} & 5.6 \\
\hline IKD & 0.654 & {$[0.040,0.904]$} & 3.6 & 0.876 & {$[0.397,0.971]$} & 6.9 \\
\hline \multicolumn{7}{|c|}{ Inter-session Reproducibility $\ddagger$} \\
\hline HHD & 0.909 & {$[0.695,0.976]$} & 6.0 & 0.854 & {$[0.297,0.966]$} & 5.2 \\
\hline LSMD & 0.959 & {$[0.852,0.989]$} & 11.3 & 0.938 & {$[0.725,0.985]$} & 9.7 \\
\hline IKD & 0.916 & {$[0.700,0.978]$} & 7.5 & 0.933 & {$[0.738,0.983]$} & 9.1 \\
\hline
\end{tabular}

HHD = Hand-held dynamometry; LSMD = Limb strength measurement device; IKD = fixed-isokinetic (isometric) dynamometry; ICC = Intra-class correlation coefficient for consistency ${ }^{\dagger}\left(\operatorname{ICC}_{\text {con }}(3,1)\right)$ and absolute agreement $\ddagger\left(\operatorname{ICC}_{\mathrm{aa}}(2,1)\right) ; \mathrm{CI}_{95}=95 \%$ confidence interval (lower bound, LB and upper bound, UB) on the ICC; $d_{C o h e n}=$ Cohen's d-index for effect size.

\subsubsection{Inter-Rater Reproducibility}

This analysis used session I and session II data measured by rater A and rater B, respectively, on a sub-sample of participants (group $2, \mathrm{n}_{2}=10$ ) measured by both raters. Reproducibility results using the $\operatorname{ICC}_{\mathrm{aa}}(2,1)$ model are shown in Table 4. ICC values for this analysis were lower than for intra-rater results. Although flexor assessment had good inter-rater reliability with all three devices with ICC values $>0.85$, for extensor strength measurement only the LSMD achieved good results (ICC $=0.897$ ), whereas the HHD and IKD results were poor (ICC $=0.575$ and 0.654 respectively).

\subsubsection{Inter-Session Reproducibility}

This analysis used session I and session II data measured only by rater A, on a sub-sample of participants (group 1, $n_{1}=10$ ) measured by this rater in both sessions. Reproducibility between sessions for a single rater using the $\operatorname{ICC}_{\mathrm{aa}}(2,1)$ model are shown in Table 4 . Inter-session reproducibility results were excellent (ICC > 0.90) for all measures except flexion strength with the HHD, which had lower but good $($ ICC $=0.854)$ reproducibility.

\subsubsection{Post-Hoc Analysis of Reproducibility Results}

Simple main effects were examined in the $\operatorname{ICC}_{\mathrm{aa}}(2,1)$ model for reproducibility across sessions (group 1) and raters (group 2). These data are shown in Table 5. In terms of between-session differences, only the HHD for flexor strength was statistically significant $(p=0.017)$. Between-rater differences were more severe with a significant difference for HHD in extension $(p=0.001)$, and IKD in both extension $(p=0.025)$ and flexion $(p=0.023)$. 
Table 5. Significance of the between-sessions and between-raters component of variance for ICC tests.

\begin{tabular}{|c|c|c|c|c|c|c|}
\hline & & & \multicolumn{2}{|c|}{ Extension } & \multicolumn{2}{|c|}{ Flexion } \\
\hline \multicolumn{3}{|c|}{$\mathrm{n}=\mathbf{1 0}$ per Group } & $F_{j}^{+}$ & $\boldsymbol{P}_{j}{ }^{\dagger}$ & $F_{j}^{+}$ & $p_{j}^{+}$ \\
\hline Inter-session & $\exists$ & HHD & 0.9 & 0.372 & 8.4 & $0.017^{*}$ \\
\hline $\operatorname{ICC}_{a a}(2,1)$ & $\dot{s}$ & LSMD & 1.3 & 0.283 & 4.5 & 0.064 \\
\hline [Group 1] & 5 & IKD & 0.1 & 0.721 & 3.3 & 0.104 \\
\hline Inter-rater & $\infty$ & HHD & 24.5 & 0.001 * & 1.4 & 0.260 \\
\hline $\operatorname{ICC}_{a a}(2,1)$ & $\dot{>}$ & LSMD & 0.6 & 0.470 & 0.2 & 0.634 \\
\hline [Group 2] & $\varangle$ & IKD & 7.3 & 0.025 * & 7.4 & 0.023 * \\
\hline
\end{tabular}

HHD = Hand-held dynamometry; LSMD = Limb strength measurement device; IKD = fixed-isokinetic (isometric) dynamometry; $\operatorname{ICC}_{\mathrm{aa}}(2,1)=$ Intra-class correlation coefficient, model 2 for absolute agreement. S I = Session one testing; $\mathrm{SII}=$ Session two testing; $\mathrm{A}=$ Rater $\mathrm{A} ; \mathrm{B}=$ Rater $\mathrm{B} ;{ }^{\dagger} F$-statistic and $p$-value for significance; ${ }^{*}$ Significant effect with $\alpha=0.05$.

\subsection{Validity Analysis}

\subsubsection{Criterion Validity}

This analysis used session I data for the LSMD and the IKD devices. For the purpose of validation, the LSMD was considered the experimental measurement and IKD considered the criterion standard (i.e., 'gold standard') measurement. Model IIA regression results are shown in Figure 5.
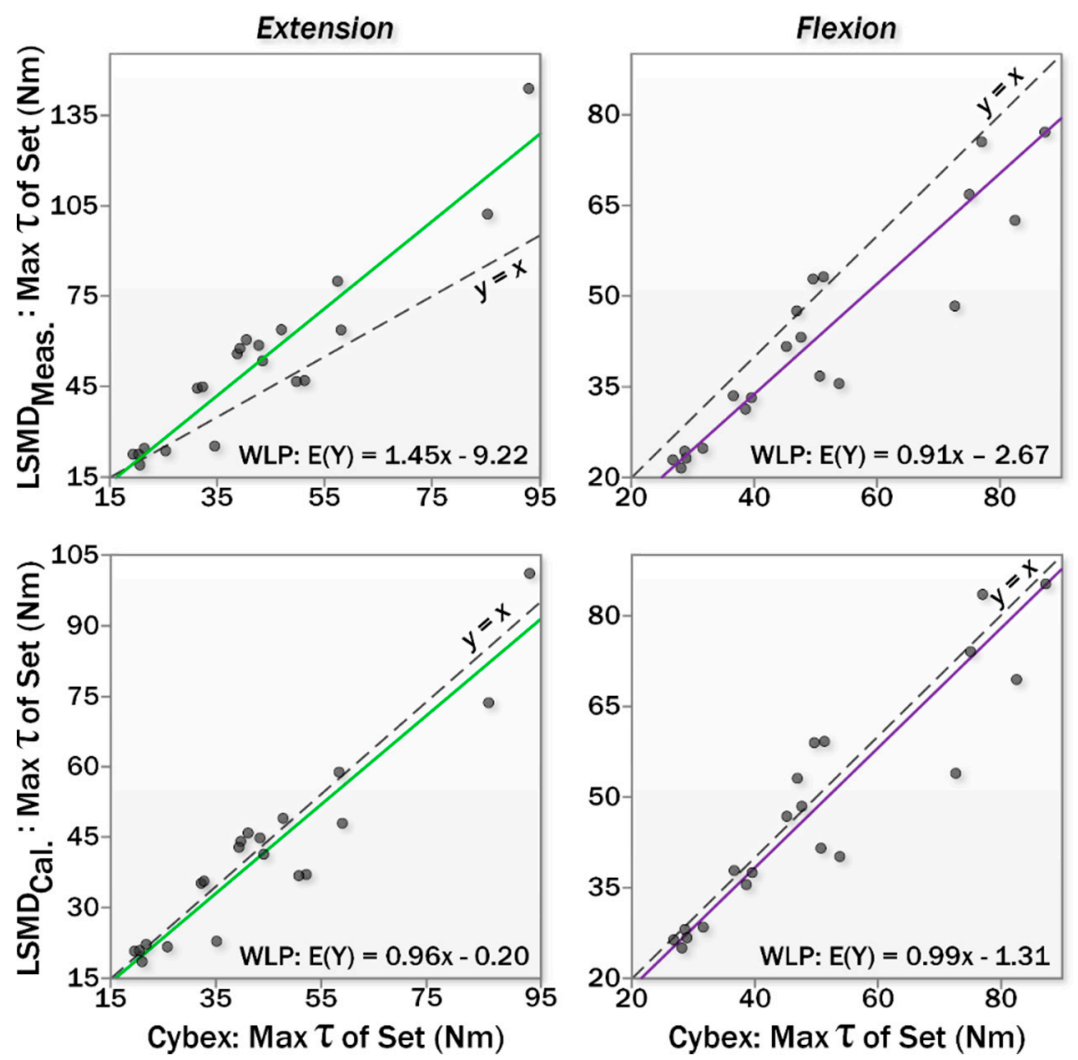

Figure 5. Weighted least squares regressions for LSMD versus IKD for extensors (left) and flexors (right), as measured (top) and after post-hoc calibration (bottom).

The plots show that the LSMD over-predicts elbow extensor strength and under-predicts elbow flexor strength. Analysis of the regression coefficients for LSMD versus IKD showed a significant proportional bias effect for both flexion and extension, as shown in Table 6. 
Table 6. Summary of WLP regression results for Session I and Session II.

\begin{tabular}{|c|c|c|c|c|c|c|c|c|c|}
\hline \multicolumn{3}{|c|}{ Comparison } & $r^{2}$ & $a$ & $\mathrm{CI}_{95}$ for $a^{\prime}$ & $p_{a}^{+}$ & $b$ & $\mathrm{CI}_{95}$ for $b$ & $p_{b}{ }^{\dagger}$ \\
\hline \multicolumn{10}{|l|}{ Session I } \\
\hline \multirow{2}{*}{\multicolumn{2}{|c|}{$\begin{array}{c}\text { LSMD } \\
\text { vs. } \\
\text { IKD }\end{array}$}} & Ext. & 0.810 & -9.022 & {$[-26.627,8.583]$} & 0.296 & 1.513 & {$[0.870,2.155]$} & $<0.000^{*}$ \\
\hline & & Flex. & 0.884 & -1.459 & {$[-8.005,5.087]$} & 0.645 & 0.922 & {$[0.736,1.108]$} & $<0.000$ * \\
\hline \multicolumn{10}{|l|}{ Session II } \\
\hline \multirow{4}{*}{$\begin{array}{l}\text { LSMD } \\
\text { vs. } \\
\text { IKD }\end{array}$} & \multirow[b]{2}{*}{ Measured } & Ext. & 0.885 & -9.220 & {$[-21.973,3.534]$} & 0.146 & 1.451 & {$[1.006,1.895]$} & $<0.000 *$ \\
\hline & & Flex. & 0.855 & -2.666 & {$[-10.856,5.523]$} & 0.503 & 0.912 & {$[0.700,1.124]$} & $<0.000 *$ \\
\hline & \multirow{2}{*}{ Calibrated } & Ext. & 0.885 & -0.203 & {$[-7.380,6.974]$} & 0.953 & 0.964 & {$[0.732,1.196]$} & $<0.000 *$ \\
\hline & & Flex. & 0.855 & -1.315 & {$[-8.841,6.212]$} & 0.718 & 0.990 & {$[0.806,1.174]$} & $<0.000 *$ \\
\hline
\end{tabular}

LSMD = Limb strength measurement device; IKD = fixed-isokinetic (isometric) dynamometry; $\mathrm{CI}_{95}=95 \%$ confidence interval on the indicated coefficient; ${ }^{\dagger} p$-value for significance; * Significant effect with $\alpha=0.05$.

\subsubsection{Calibration Using the Criterion Standard}

Regression coefficients from the above analysis of LSMD and IKD session I data were used to derive calibration formulas for LSMD elbow extensor and flexor measurement.

$$
\begin{aligned}
\tau_{e}^{C a l} & =\frac{1}{1.513}\left(\tau_{e}+9.022\right) \\
\tau_{f}^{C a l} & =\frac{1}{0.922}\left(\tau_{f}+1.459\right)
\end{aligned}
$$

where $\tau_{e}$ and $\tau_{f}$ are the measured extensor and flexor torques, respectively, and $\tau_{e}{ }^{\mathrm{Cal}}$ and $\tau_{f}^{\mathrm{Cal}}$ are the corrected torques.

Finally, Equations (3) and (4) were applied to LSMD data from session II and compared to IKD data for session II. Results are shown in terms of regression coefficients in Table 6 and measured torque values in Table 7. Before calibration, the LSMD overestimated extension strength by $21 \%$ and underestimated flexion strength by $14 \%$. Calibration narrowed these effects, with the LSMD underestimating strength by $3 \%$ of the IKD measurement in both flexion and extension, falling within the random error measured during repeated measurements with the criterion device. LoA plots for extensor and flexor strength measurements are shown in Figure 6.
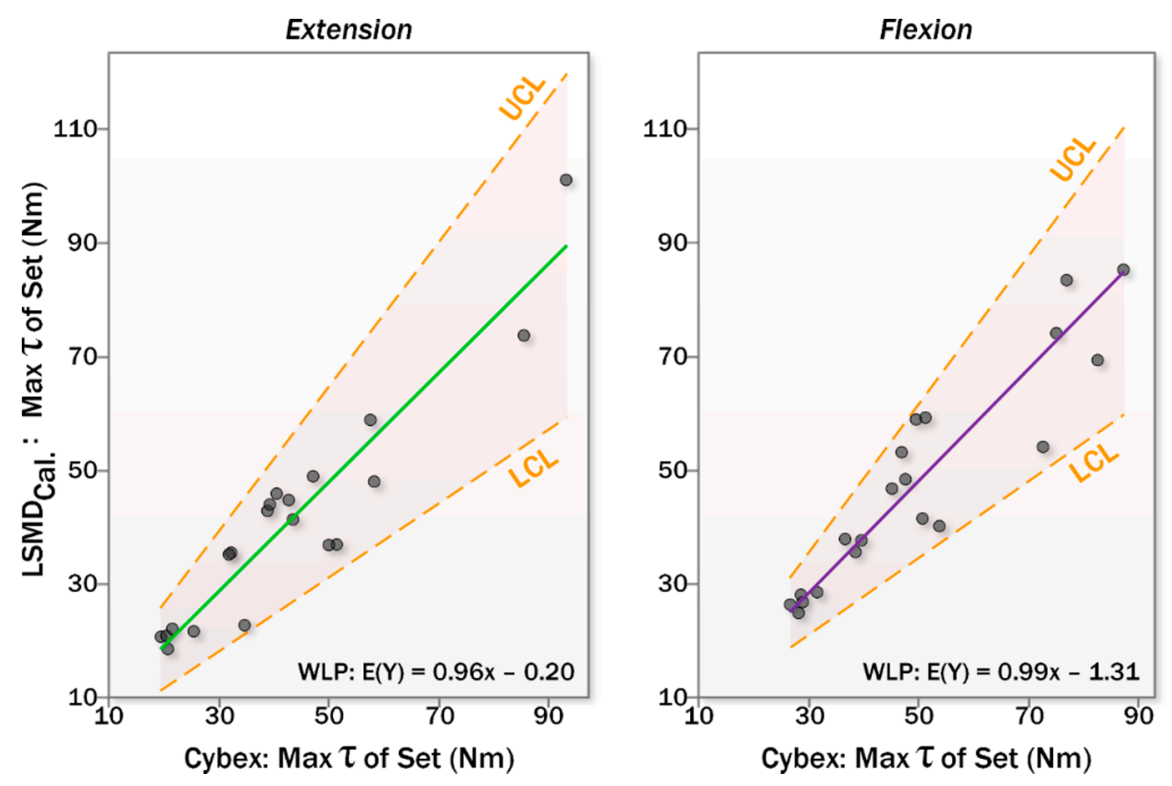

Figure 6. Limits of agreement plots for LSDM relative to IKD for extensors (left) and flexors (right). 
Table 7. Effect of calibration on session II LSMD torque data.

\begin{tabular}{cccccccc}
\hline & Muscle & \multicolumn{2}{c}{$\tau(\mathbf{N m})$} & \multicolumn{3}{c}{$\Delta \tau$ (IKD-LSMD) (Nm) } \\
\hline & Group & IKD & LSMD & Mean & $\mid$ min| & $\mid$ max & $s$ \\
\hline As & Ext. & 42.6 & 53.1 & -10.4 & 1.5 & 50.9 & 13.4 \\
Measured & Flex. & 49.9 & 42.9 & 7.0 & 0.7 & 24.2 & 7.2 \\
\hline \multirow{2}{*}{ Calibrated } & Ext. & 42.6 & 41.1 & 1.6 & 0.5 & 14.3 & 6.9 \\
& Flex. & 49.9 & 48.1 & 1.8 & 0.2 & 18.5 & 7.2 \\
\hline
\end{tabular}

LSMD = Limb strength measurement device; IKD = fixed-isokinetic (isometric) dynamometry; $s$ = Standard deviation; $\tau=$ Torque.

\section{Discussion}

Reliable and valid measurement of muscle strength is paramount for clinical decision making $[5,6]$. However, the literature shows few advances in strength assessment beyond the ubiquitous HHD and IKD devices introduced more than three decades ago. The LSMD was originally designed as a component of the BioTone ${ }^{\mathrm{TM}}$ system, intended for muscle tone assessment in the clinic of persons with upper motor neuron syndrome resulting from neurological injury or disease [47]. More broadly, the LSMD has potential for assessing elbow (and knee) strength in any population, for any number of compelling reasons. Whether quantifying strength of seniors for prescribing interventions, or evaluating contractile strength in athletes following sport-related injuries, the LSMD could provide a portable alternative to fixed-IKD. Furthermore, while the HHD is a clinically accepted approach to assessing strength, the superior reliability of the LSMD could improve the quality of strength assessments used in practice and in clinical trials of new treatments and drugs.

The purpose of this study was to evaluate the reliability and validity of the LSMD for these broader applications. This study focused on healthy adults as a first step to establish the quality, and limitations, of the measurement capability of the LSMD.

\subsection{Reliability of the LSMD}

To put the reliability results into context, recommended ICC cut-offs from the published literature were examined. Schrama et al. [3] provided a set of recommended cut-offs for ICC results in strength measurement studies that were specific to the type of reliability being measured. These cut-offs are shown below in Table 8. The guidelines of Lohr et al. [48] and Kottner et al. [49] were used to supplement the recommendations of Schrama et al. by providing a clinical viewpoint on minimal standards for ICCs. The analysis of van Trijffel et al. [50] provided a logical set of boundaries for judging the meta-reliability of a study that lists many ICC values associated with a single protocol.

Table 8. Recommended cut-off values for ICC acceptability.

\begin{tabular}{cccc}
\hline \multicolumn{2}{c}{ Reliability } & Recommended Cut-Off & Source(s) \\
\hline Intra-rater & $\operatorname{ICC}_{\mathrm{con}}(3,1)$ & $>0.90$ & {$[3,48,49]$} \\
Inter-rater & $\operatorname{ICC}_{\mathrm{aa}}(2,1)$ & $>0.75$ & {$[3,48]$} \\
Inter-session & $\operatorname{ICC}_{\mathrm{aa}}(2,1)$ & $>0.75$ & {$[3,48]$} \\
\multicolumn{2}{l}{ Overall reliability of the study } & $\geq 75 \%$ of listed $\mathrm{ICC}$ values are $>0.75$ & {$[50]$} \\
\hline
\end{tabular}

Note: where more than one cut-off value was recommended in the literature, the most stringent value was chosen.

While all three devices passed the van Trijffel et al. meta-reliability standards [50], the LSMD was the only device to exceed the ICC cut-offs in all testing conditions. In fact, the ICC values for the LSMD ranked highest for all but two of the six experimental conditions (see Table 4). It can be concluded that, despite any deficits in accuracy due to small deflections of the device, measurement of MVIC strength with the LSMD was repeatable and reproducible. This makes sense given that the LSMD structure (3-point contact) must equilibrate on the arm of the user arm during muscle contractions. 
Provided that the arm geometry of the user does not change between assessments, one would expect this equilibrium point to be consistent across assessments.

The IKD and the HHD devices also performed acceptably across each reliably metric. These data agree with the HHD reliability data presented in studies by Visser et al. [51], Bohannon [52] and Aufsesser et al. [53]. Similarly, the Cybex fixed-IKD reliability data agree with the intra- and rater reliabilities reported by Mathur et al. [54], Ekstrand et al. [55], and Stratford and Balsor [56].

The post-hoc analysis of inter-session and inter-rater reproducibility (Table 7) provided a better understand the reasons for observing lower reproducibility of the HHD and IKD for some measures. The between-raters data in Table 7 revealed a significant between-raters difference for the IKD in both extension and flexion. These data suggest that, for the Cybex IKD device, the source of the inter-rater variability is independent of the type of elbow motion. It can be concluded that alignment of the elbow joint in the Cybex machine was most likely responsible, given that it is the only source of variability directly involving the rater. While both raters were trained with the same instructional content and hands-on practice, clearly even small differences in alignment with the Cybex motor axis can result in less reproducible results, reported by others [21,57]. The ability of the LSMD to self-equilibrate likely explains why rater variability in aligning the user with the device was less influential.

While comparing reliability of the LSMD to the field 'gold standard' (IKD) is important, the reliability performance of the LSMD was also examined relative to the HDD. Although manual muscle tests are often the choice of test in the clinic [58], HHD is by far the most ubiquitous form of objective strength assessment in the clinic and research laboratory, and is known to be susceptible to variability across rater physical strength/size and skill level $[10,34,59]$. Both raters in this study were novice, though equally trained, assessors. As shown in Table 7, difference between-raters was observed for the HHD elbow extensor torque, and difference between sessions was noted for HHD elbow flexion torque.

The between-rater difference was likely due in part to a protocol deviation that occurred, reflecting the novice level of the two raters. One rater started with a break-test rather than a make test, which was then maintained for consistency. This would account for observed difference in elbow extension, since the ability to stabilize against strong is different between the make and break tests [56]. Finally it should be noted that a few participants in the study were, by all accounts, stronger than the raters, which no doubt contributed to the higher variability across raters and sessions $[13,60]$.

\subsection{Validity of the LSMD}

Although excellent reliability was demonstrated for the LSMD, the validity of the device when tested against the criterion standard was less impressive before calibration. As shown in Table 3 and Figure 5, the LSMD over-predicted extensor muscle strength, and under-predicted flexor muscle strength. Fortunately, however, the disagreement between LSMD and criterion standard was a linear function of strength magnitude and was easily modelling using the WLP regression results. After applying these corrections to the measured LSMD data from a different session (data not used to create the calibration equations), the difference between LSMD and criterion standard disappeared, lending credence to this approach for improving the validity of both extensor and flexor muscle strength measurements.

There are some important implications of these findings. As noted above, the sample studied was from a population of university students and staff, representing a young and healthy population with higher than average strength compared to the general population (c.f. study by Hogrel et al. [61]) but demonstrating heteroscedastistic variability. Therefore, the effect of device deformation was driven by the strongest participants in the study. In clinical studies using the LSMD [30,31,62], participants were not likely strong enough to deform the LSMD. Nevertheless, the need for a correction at all remains a design concern.

To further explore this characteristic, Figure 7 shows a force diagram of the LSMD in its extensor and flexor configurations. Assuming the force through the wrist cuff remains perpendicular to 
the forearm, the force vectors through the proximal and distal upper arm cuffs provide insight into how the device self-stabilizes, and how it may in some circumstance negatively impact the measurement accuracy.

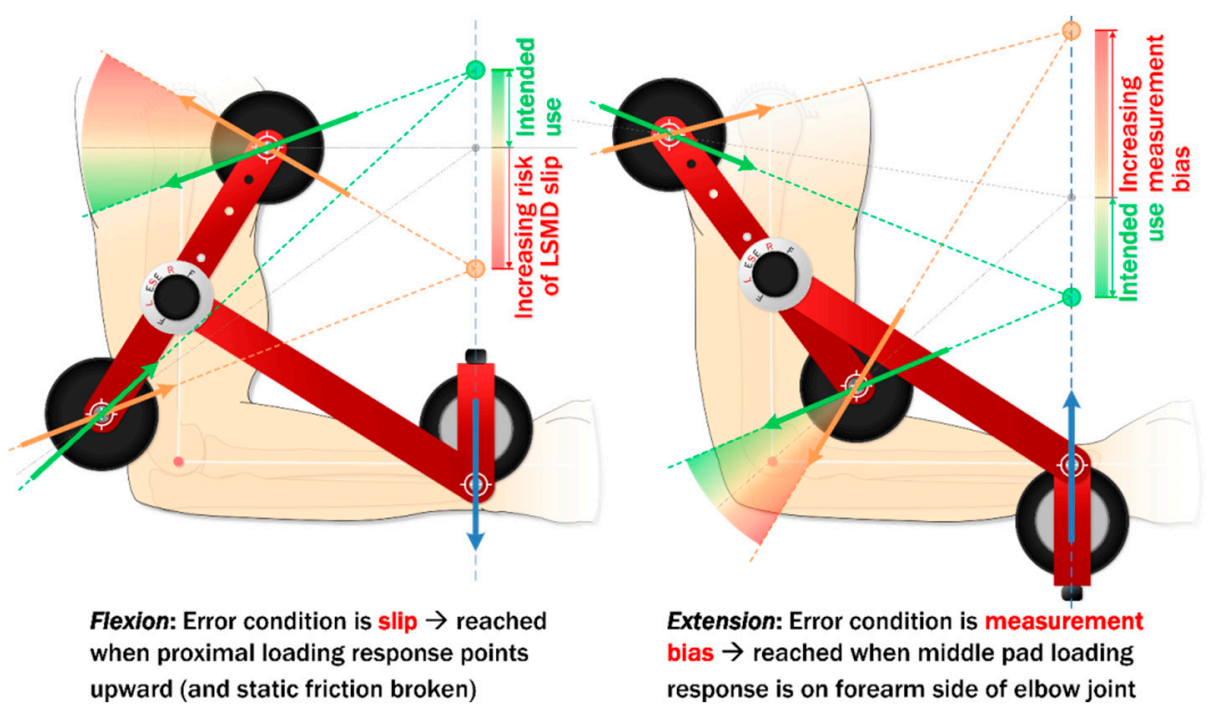

Figure 7. Biomechanical explanation of device measurement fidelity limitations.

The right side of Figure 7 shows the device in extension strength configuration. Since the device represents a simple 3-force body, all three forces must pass through a common point in order for equilibrium to be achieved. For each configuration, vectors are shown at two extremes, where the green vectors show the desired force directions, and the orange vectors show the potentially problematic force directions.

For extension it can be seen that, depending on the direction of force through the posterior-proximal cuff, the force through the anterio-distal cuff located at the crux of the elbow could transmit loads to the forearm, thus inflating the measured elbow torque (shown by the orange vectors). For flexion it can also be seen that variability in anterio-proximal cuff force vector has a potential effect. If force directions are oriented as shown with the orange vectors, the net vertical force is directed upward, potentially causing the device to migrate upward on the arm and causing apparent under-prediction of flexor strength. It is likely that these biomechanical factors play a role in device measurement fidelity, and are currently the subject of further study and design by the authors.

\subsection{Limitations}

There are some important limitations of the study to point out. Although the full cohort was of sufficient sample size $(n=20)$, the sub-groups used to test reproducibility were smaller $(n=10)$, making them more susceptible to sampling bias. Nevertheless, ICC values agreed well with the literature and any aberrant findings were explainable.

The study only included healthy adult participants. Indeed, the intent of the device is for assessing strength across the population. This includes seniors and clinical populations that may have different underlying force generating characteristics, and thus reliability can not necessarily be extrapolated from the data in the present study. Future studies will be needed to evaluate the psychometrics of the LSMD in specific populations where applications exist. However, the data given in Figure 6 provides sufficient information for a clinician to infer the suitability of the LSMD for their individual needs.

This study only included the upper-extremity LSMD for the elbow joint. Although it is likely that the LSMD knee device (discussed in [30]) behaves similar to the elbow device, since it is essentially a scaled-up version of the arm model, future studies will need to apply the testing protocol to evaluate reliability and validity of the LSMD for lower-extremity assessment. 


\section{Conclusions}

The reliability (repeatability and reproducibility) of the LSMD was found to be comparable to established commercial devices for measurement of elbow flexor and extensor strength in healthy adults. Validity of the LSMD was found to be acceptable when measurements were calibrated against the accepted gold standard IKD measurement. It is concluded that a calibrated wearable device such as the LSMD can be used to get reliable and valid measurements of elbow strength.

Author Contributions: M.B. wrote the manuscript draft, designed and executed the experiment, and analyzed the data. A.S. managed the BioTone project, provided hardware and software support, and assisted with manuscript preparation and data collection. C.A.M. led the development of the LSMD, conceived of and acquired funding for the project, and assisted with manuscript preparation and data analysis. All authors have read and agreed to the published version of the manuscript.

Funding: The project was funded through the Atlantic Canada Opportunities Agency, Atlantic Innovation Fund, Project \# 195180.

Acknowledgments: The authors thank the students and staff of the Andrew and Marjorie McCain Human Performance Laboratory and the Institute of Biomedical Engineering.

Conflicts of Interest: The authors declare no conflicts of interest.

\section{References}

1. Osternig, L.R. Isokinetic dynamometry: Implications for muscle testing and rehabilitation. Exerc. Sport Sci. Rev. 1986, 14, 45-80. [CrossRef] [PubMed]

2. Baltzopoulos, V.; Brodie, D. Isokinetic dynamometry. Applications and limitations. Sport. Med. 1989, 8, 101-116. [CrossRef] [PubMed]

3. Schrama, P.P.; Stenneberg, M.S.; Lucas, C.; van Trijffel, E. Intraexaminer reliability of hand-held dynamometry in the upper extremity: A systematic review. Arch. Phys. Med. Rehabil. 2014, 95, 2444-2469. [CrossRef] [PubMed]

4. Bourne, M.N.; Opar, D.A.; Williams, M.D.; Shield, A.J. Eccentric knee flexor strength and risk of hamstring injuries in rugby union: A prospective study. Am. J. Sports Med. 2015, 43, 2663-2670. [CrossRef]

5. Keating, J.L.; Matyas, T.A. The influence of subject and test design on dynamometric measurements of extremity muscles. Phys. Ther. 1996, 76, 866-889. [CrossRef]

6. Marx, R.G.; Menezes, A.; Horovitz, L.; Jones, E.C.; Warren, R.F. A comparison of two time intervals for test-retest reliability of health status instruments. J. Clin. Epidemiol. 2003, 56, 730-735. [CrossRef]

7. Harris-Love, M.; Benson, K.; Leasure, E.; Adams, B.; McIntosh, V. The Influence of Upper and Lower Extremity Strength on Performance-Based Sarcopenia Assessment Tests. J. Funct. Morphol. Kinesiol. 2018, 3, 53. [CrossRef]

8. Davies, G.J.; McCarty, E.; Provencher, M.; Manske, R.C. ACL Return to Sport Guidelines and Criteria. Curr. Rev. Musculoskelet. Med. 2017, 10, 307-314. [CrossRef]

9. Bohannon, R.W. Considerations and Practical Options for Measuring Muscle Strength: A Narrative Review. Biomed Res. Int. 2019, 2019, 1-10. [CrossRef]

10. Bohannon, R.W. Hand-held dynamometry: Factors influencing reliability and validity. Clin. Rehabil. 1997, 11, 263-264.

11. Wikholm, J.B.; Bohannon, R.W. Hand-held dynamometer measurements: Tester strength makes a difference. J. Orthop. Sports Phys. Ther. 1991, 13, 191-198. [CrossRef] [PubMed]

12. Stone, C.A.; Bríd, N.; Lawlor, P.G.; Kenny, R.A. Hand-held dynamometry: Tester strength is paramount, even in frail populations. J. Rehabil. Med. 2011, 43, 808-811. [CrossRef] [PubMed]

13. Byl, N.N.; Richards, S.; Asturias, J. Intrarater and interrater reliability of strength measurements of the biceps and deltoid using a hand held dynamometer. J. Orthop. Sports Phys. Ther. 1988, 9, 395-398. [CrossRef] [PubMed]

14. Kelln, B.M.; McKeon, P.O.; Gontkof, L.M.; Hertel, J. Hand-held dynamometry: Reliability of lower extremity muscle testing in healthy, physically active, young adults. J. Sport Rehabil. 2008, 17, 160-170. [CrossRef]

15. Bohannon, R.W. Intertester reliability of hand-held dynamometry: A concise summary of published research. Percept. Mot. Skills 1999, 88, 899-902. [CrossRef]

16. Brinkrnann, J.R. Comparison of a hand-held and fixed dynamometer in measuring strength of patients with neuromuscular disease. J Orthop Sport. Phys Ther. 1994, 19, 100-104. [CrossRef] 
17. Thorborg, K.; Bandholm, T.; Hölmich, P. Hip- and knee-strength assessments using a hand-held dynamometer with external belt-fixation are inter-tester reliable. Knee Surg Sport. Traumatol Arthrosc. 2013, 21, 550-555. [CrossRef]

18. Gagnon, D.; Nadeau, S.; Gravel, D.; Robert, J.; Belanger, D.; Hilsenrath, M. Reliability and validity of static knee strength measurements obtained with a chair-fixed dynamometer in subjects with hip or knee arthroplasty. Arch. Phys. Med. Rehabil. 2005, 86, 1998-2008. [CrossRef]

19. Bandy, W.D.; McLaughlin, S. Intramachine and intermachine reliability for selected dynamic muscle performance tests. J. Orthop. Sports Phys. Ther. 1993, 18, 609-613. [CrossRef]

20. Reed, R.L.; Hartog, R.D.; Yochum, K.; Pearlmutter, L.; Ruttinger, A.C.; Mooradian, A.D. A comparison of hand-held isometric strength measurement with isokinetic muscle strength measurement in the elderly. J. Am. Geriatr. Soc. 1993, 41, 53-56. [CrossRef]

21. Rothstein, J.M.; Lamb, R.L.; Mayhew, T.P. Clinical uses of isokinetic measurements. Critical issues. Phys. Ther. 1987, 67, 1840-1844. [CrossRef] [PubMed]

22. Gleeson, N.P.; Mercer, T.H. The utility of isokinetic dynamometry in the assessment of human muscle function. Sport. Med. 1996, 21, 18-34. [CrossRef] [PubMed]

23. Stark, T.; Walker, B.; Phillips, J.K.; Fejer, R.; Beck, R. Hand-held dynamometry correlation with the gold standard isokinetic dynamometry: A systematic review. PMER 2011, 3, 472-479.

24. Reinking, M.F.; Bockrath-Pugliese, K.; Worrell, T.; Kegerreis, R.L.; Miller-Sayers, K.; Farr, J. Assessment of quadriceps muscle performance by hand-held, isometric, and isokinetic dynamometry in patients with knee dysfunction. J. Orthop. Sport. Phys. Ther. 1996, 24, 154-159. [CrossRef] [PubMed]

25. Cockburn, E.; Hayes, P. Test-retest reliability of isokinetic concentric knee extension and flexion. Br. J. Sports Med. 2010, 44, i26. [CrossRef]

26. Kannus, P. Isokinetic evaluation of muscular performance: Implications for muscle testing and rehabilitation. Int. J. Sports Med. 1994, 15, S11-8. [CrossRef]

27. Lund, H.H.; Søndergaard, K.; Zachariassen, T.; Christensen, R.; Bülow, P.; Henriksen, M.; Bartels, E.M.; Danneskiold-Samsøe, B.; Bliddal, H. Learning effect of isokinetic measurements in healthy subjects, and reliability and comparability of Biodex and Lido dynamometers. Clin. Physiol. Funct. Imaging 2005, 25, 75-82. [CrossRef]

28. Drouin, J.M.; Valovich-McLeod, T.C.; Shultz, S.J.; Gansneder, B.M.; Perrin, D.H. Reliability and validity of the Biodex system 3 pro isokinetic dynamometer velocity, torque and position measurements. Eur. J. Appl. Physiol. 2004, 91, 22-29.

29. Landry, J.; Sexton, A.; Hughes, G.; McGibbon, C.A. Limb Strength Measurement Device. U.S. Patent US9,028,433, 12 May 2015.

30. McGibbon, C.A.; Sexton, A.; Hughes, G.; Wilson, A.; Jones, M.; O'Connell, C.; Parker, K.; Adans-Dester, C.; O'Brien, A.; Bonato, P. Evaluation of a toolkit for standardizing clinical measures of muscle tone. Physiol. Meas. 2018, 39, 085001. [CrossRef]

31. McGibbon, C.A.; Sexton, A.; Jones, M.; O'Connell, C.A.; O'Connell, C. Elbow spasticity during passive stretch-reflex: Clinical evaluation using a wearable sensor system. J. Neuroeng. Rehabil. 2013, 10, 61. [CrossRef]

32. Kollock, R.O.; Onate, J.A.; Lunen, B. Van The reliability of portable fixed dynamometry during hip and knee strength assessments. J. Athl. Train. 2010, 45, 349-356. [CrossRef] [PubMed]

33. Schless, S.H.; Desloovere, K.; Aertbeliën, E.; Molenaers, G.; Huenaerts, C.; Bar-On, L. The intra- and inter-rater reliability of an instrumented spasticity assessment in children with cerebral palsy. PLoS ONE 2015, 10, e0131011. [CrossRef] [PubMed]

34. Scott, D.A.; Bond, E.Q.; Sisto, S.A.; Nadler, S.F. The intra- and interrater reliability of hip muscle strength assessments using a handheld versus a portable dynamometer anchoring station. Arch. Phys. Med. Rehabil. 2004, 85, 598-603. [CrossRef]

35. Ford-Smith, C.D.; Wyman, J.F.; Elswick, R.K. Reliability of stationary dynamometer muscle strength testing in community-dwelling older adults. Arch. Phys. Med. Rehabil. 2001, 82, 1128-1132. [CrossRef] [PubMed]

36. Souza, L.A.; Martins, J.C.; Teixeira-Salmela, L.F.; Lara, E.M.; Moura, J.B.; Aguiar, L.T.; de Morais Faria, C.D. Validity and reliability of the modified sphygmomanometer test to assess strength of the lower limbs and trunk muscles after stroke. J Rehabil Med. 2014, 46, 620-628. [CrossRef] [PubMed] 
37. Lu, T.W.; Hsu, H.C.; Chang, L.Y.; Chen, H.L. Enhancing the examiner's resisting force improves the reliability of manual muscle strength measurements: Comparison of a new device with hand-held dynamometry. J. Rehabil. Med. 2007, 39, 679-684. [CrossRef]

38. HUMAC NORM Brochure; Computer Sports Medicine Inc.: Stoughton, MA, USA, 2009.

39. Hoggan Scientific Inc. Ergo Force Gauges, Medical Force Gauges \& Sensors - microFET2. Available online: https://hogganscientific.com/product/microfet2-muscle-tester-digital-handheld-dynamometer/ (accessed on 17 June 2020).

40. Bohannon, R.W. Test-retest reliability of hand-held dynamometry during a single session of strength assessment. Phys. Ther. 1986, 66, 206-209. [CrossRef]

41. HUMAC/NORM Testing and Rehabilitation System: User Guide (Model 770); Computer Sports Medicine Inc.: Stoughton, MA, USA, 2009.

42. Matheson, L.; Mooney, V.; Caiozzo, V.; Jarvis, G.; Pottinger, J.; DeBerry, C.; Backlund, K.; Klein, K.; Antoni, J. Effect of instructions on isokinetic trunk strength testing variability, reliability, absolute value, and predictive validity. Spine (Phila. Pa. 1976) 1992, 17, 914-921. [CrossRef]

43. Shrout, P.E.; Fleiss, J.L. Intraclass correlations: Uses in assessing rater reliability. Psychol. Bull. 1979, 86, 420-428. [CrossRef]

44. Bland, J.M.; Altman, D.G. Statistical methods for assessing agreement between two methods of clinical measurement. Lancet 1986, 1, 307-310. [CrossRef]

45. Ludbrook, J.J. Confidence in Altman-Bland plots: A critical review of the method of differences. Clin. Exp. Pharmacol. Physiol. 2010, 37, 143-149. [CrossRef] [PubMed]

46. Ludbrook, J.J. Comparing methods of measurements. Clin. Exp. Pharmacol. Physiol. 1997, 24, $193-203$. [CrossRef] [PubMed]

47. Miczak, K.; Padova, J. Muscle Overactivity in the Upper Motor Neuron Syndrome: Assessment and Problem Solving for Complex Cases: The Role of Physical and Occupational Therapy. Phys. Med. Rehabil. Clin. N. Am. 2018, 29, 529-536. [CrossRef]

48. Lohr, K.N.; Aaronson, N.K.; Alonso, J.; Audrey, B.M.; Patrick, D.L.; Perrin, E.B.; Roberts, J.S. Evaluating quality-of-life and health status instruments: Development of scientific review criteria. Clin. Ther. 1996, 18, 979-992. [CrossRef]

49. Kottner, J.; Audigé, L.; Brorson, S.; Donner, A.; Gajewski, B.J.; Hróbjartsson, A.; Roberts, C.; Shoukri, M.; Streiner, D.L. Guidelines for reporting reliability and agreement studies (GRRAS) were proposed. J. Clin. Epidemiol. 2011, 64, 96-106. [CrossRef]

50. van Trijffel, E.E.; van de Pol, R.J.; Oostendorp, R.A.; Lucas, C. Inter-rater reliability for measurement of passive physiological movements in lower extremity joints is generally low: A systematic review. J. Physiother. 2010, 56, 223-235. [CrossRef]

51. Visser, J.; Mans, E.; de Visser, M.; van den Berg-Vos, R.M.; Franssen, H.; de Jong, J.M.; van den Berg, L.H.; Wokke, J.H.; de Haan, R.J. Comparison of maximal voluntary isometric contraction and hand-held dynamometry in measuring muscle strength of patients with progressive lower motor neuron syndrome. Neuromuscul. Disord. 2003, 13, 744-750. [CrossRef]

52. Bohannon, R.W. Reference values for extremity muscle strength obtained by hand-held dynamometry from adults aged 20 to 79 years. Arch. Phys. Med. Rehabil. 1997, 78, 26-32. [CrossRef]

53. Aufsesser, P.M.; Horvat, M.; Austin, R. The reliability of hand held muscle testers with individuals with spinal cord injury. Clin Kinesiol. 2003, 57, 71-75.

54. Mathur, S.; Makrides, L.; Hernandez, P. Test-retest reliability of isometric and isokinetic torque in patients with chronic obstructive pulmonary disease. Physiother. Canada 2004, 56, 94-101. [CrossRef]

55. Ekstrand, E.; Lexell, J.; Brogårdh, C. Isometric and isokinetic muscle strength in the upper extremity can be reliably measured in persons with chronic stroke. J. Rehabil. Med. 2015, 47, 706-713. [CrossRef] [PubMed]

56. Stratford, P.W.; Balsor, B.E. A comparison of make and break tests using a hand-held dynamometer and the Kin-Com. J. Orthop. Sports Phys. Ther. 1994, 19, 28-32. [CrossRef] [PubMed]

57. Caruso, J.; Brown, L.; Tufano, J. The reproducibility of isokinetic dynamometry data. Isokinet. Exerc. Sci. 2012, 20, 239-253. [CrossRef]

58. Jensen, A.M. Estimating the prevalence of use of kinesiology-style manual muscle testing: A survey of educators. Adv. Integr. Med. 2015, 2, 96-102. [CrossRef] 
59. Wadsworth, C.; Nielsen, D.H.; Corcoran, D.S.; Phillips, C.E.; Sannes, T.L. Interrater reliability of hand-held dynamometry: Effects of rater gender, body weight, and grip strength. J. Orthop. Sports Phys. Ther. 1992, 16, 74-81. [CrossRef] [PubMed]

60. Kilmer, D.D.; McCrory, M.A.; Wright, N.C.; Rosko, R.A.; Kim, H.R.; Aitkens, S.G. Hand-held dynamometry reliability in persons with neuropathic weakness. Arch. Phys. Med. Rehabil. 1997, 78, 1364-1368. [CrossRef]

61. Hogrel, J.Y.; Payan, C.A.; Ollivier, G.; Tanant, V.; Attarian, S.; Couillandre, A.; Dupeyron, A.; Lacomblez, L.; Doppler, V.; Meininger, V.; et al. Development of a French isometric strength normative database for adults using quantitative muscle testing. Arch. Phys. Med. Rehabil. 2007, 88, 1289-1297. [CrossRef]

62. Whelan, A.; Sexton, A.; Jones, M.; O'Connell, C.; McGibbon, C.A. Predictive value of the pendulum test for assessing knee extensor spasticity. J. Neuroeng. Rehabil. 2018, 15, 68. [CrossRef]

(C) 2020 by the authors. Licensee MDPI, Basel, Switzerland. This article is an open access article distributed under the terms and conditions of the Creative Commons Attribution (CC BY) license (http://creativecommons.org/licenses/by/4.0/). 